\title{
A Preconditioned Flux Reconstruction/Correction Procedure via Reconstruction Formulation for Unsteady Low Mach Number Flows on Dynamic Unstructured Meshes
}

\author{
Lai Wang* and Meilin $\mathrm{Yu}^{\dagger}$ \\ Department of Mechanical Engineering, \\ University of Maryland, Baltimore County, Baltimore, MD 21250
}

\begin{abstract}
Preconditioning methods can significantly decrease the condition number of the linear system resulted from the discretization of compressible Navier-Stokes equations at low Mach numbers by replacing the physical acoustic wave speeds with numerical ones. In the present study, the high-order accurate flux reconstruction/correction procedure via reconstruction (FR/CPR) method with low Mach number preconditioning is used to solve Navier-Stokes equations at low Mach numbers $\left(M a \sim \mathcal{O}\left(10^{-3}\right)\right)$. The dual time stepping method is used to handle unsteady flow simulations, wherein the second-order backward differentiation formula (BDF2) is adopted to discretize the temporal derivative with respect to the physical time. A simple modification of the preconditioning formulation is proposed to deal with dynamic meshes. Numerical results of several benchmark tests have demonstrated that the preconditioned FR/CPR method works well for low Mach number flows.
\end{abstract}

\section{Introduction}

The system of compressible Navier-Stokes equations becomes stiff as the Mach number decreases towards the incompressible flow regime. At low Mach numbers, the large disparity of wave speeds which makes the system ill-conditioned, can severely degrade the convergence performance and accuracy of numerical schemes. ${ }^{1}$ Preconditioning methods have been developed to reduce the condition number of compressible flows at low Mach number flows which are almost incompressible.

Traditionally, SIMPLE-family methods, ${ }^{2,3}$ projection methods ${ }^{4}$ and artificial compressibility methods ${ }^{5}$ are used to solve incompressible or nearly incompressible flows. However, these methods do not take weak fluid compressibility into account, and therefore, cannot adequately resolve complex flows with velocities spanning both incompressible and compressible regimes. As a result, it is desirable that robust, accurate and efficient numerical schemes are developed for all speeds. In general, preconditioning methods replace the physical acoustic wave speeds with numerical ones which are at the same magnitude as the flow speeds. ${ }^{6-10}$ Thus, the stiffness of compressible equations will be released. Besides, preconditioning methods can also improve the accuracy of original compressible governing equations. It is reported by Turkel et al. in Ref. 1 that the ill-conditioning

\footnotetext{
${ }^{*}$ Graduate Student, Department of Mechanical Engineering, University of Maryland, Baltimore County, AIAA member. Email: bx58858@umbc.edu

${ }^{\dagger}$ Assistant Professor, Department of Mechanical Engineering, University of Maryland, Baltimore County, AIAA senior member. Email: mlyu@umbc.edu
} 
dissipation of the original convection equations will result in loss of accuracy. The modification of the hyperbolic part of original Euler or Navier-Stokes euqations by preconditioning enables the approximately equality of dissipations in Riemann solvers so that the accuracy can be improved. A quick review of preconditioning methods reveals that various preconditoning methods can be catalogued as three major groups. ${ }^{18}$ Turkel et al. ${ }^{1,6,11,12}$ adopted $(p, \boldsymbol{v}, d S)$ as working variables. Choi et al. ${ }^{8-10}$ chose $(p, \boldsymbol{v}, T)$ as working variables. Van Leer et al. ${ }^{7,13}$ developed a symmetric preconditioner.

While Turkel et al. ${ }^{11}$ have assessed different sets of working variables, Hauke and Hughes in Ref. 14 pointed out that the conservative incompressible formulation is well defined only for the entropy variables and the primitive variables. Primitive variables can be more accurate than entropy variables. Thus, working with primitive variables is preferred in Refs. $8-10,16,17$. Note that in Ref. 15, a finite volume method for zero Mach number flows was proposed based on conservative variables. It is still an open question on the best choice of dependent variables. In this study, $(p, \boldsymbol{v}, T)$ are adopted as working variables.

Utilizing high-order schemes with preconditioning to simulate flows at low Mach numbers has gained much interest in past years, such as discontinuous Galerkin (DG). ${ }^{17,19-21}$ In this study, the high-order FR/CPR method is further developed to solve low Mach number flows. The FR/CPR method is firstly developed by Huynh to solve convection-diffusion problems. ${ }^{22,23}$ It is then extended to solve 2D conservation laws on unstructured meshes with simplex elements by Wang and Gao. ${ }^{24}$ Vincent et al. ${ }^{25}$ have established a family of energy stable FR/CPR methods. Recently, the FR/CPR method has been used to solve incompressible flows with artificial compressibility on stationary meshes ${ }^{27}$ and moving meshes. ${ }^{26}$ To the best knowledge of the authors, the current study is the first practice to use the low Mach number preconditioned FR/CPR method to solve unsteady low Mach number flows on dynamic unstructured meshes. A simple modification of the preconditioning formulation has been proposed in the present study to deal with moving meshes. ${ }^{29}$

The remainder of the paper is organized as follows. In Section II, a brief introduction of 2D Navier-Stokes equations is presented. In Section III, the low Mach number preconditioning method used in the present study is elaborated. Section IV and V explain in detail about the spatial and temporal discretization used to handle unsteady simulation. In Section VI, we present a brief discussion about the boundary conditions. In Section VII, numerical results using the preconditioned FR/CPR methods are presented. The last section concludes the present study.

\section{Governing Equations}

The compressible Navier-Stokes equations can be written as

$$
\frac{\partial \boldsymbol{q}^{c}}{\partial t}+\frac{\partial \boldsymbol{f}_{i n v}}{\partial x}+\frac{\partial \boldsymbol{g}_{i n v}}{\partial y}=\frac{\partial \boldsymbol{f}_{v i s}}{\partial x}+\frac{\partial \boldsymbol{g}_{v i s}}{\partial y}
$$

where $\boldsymbol{q}^{c}=(\rho, \rho u, \rho v, E)^{T}$ are the conservative variables, $p=\rho R T, E=\frac{p}{\gamma-1}+\frac{1}{2} \rho\left(u^{2}+v^{2}\right), R$ is the ideal gas constant, and $\gamma$ is the specific heat ratio defined as $\gamma=C_{p} / C_{v}$. For air, $\gamma$ is set as 1.4. The component forms of $\boldsymbol{f}_{\text {inv }}, \boldsymbol{g}_{\text {inv }}, \boldsymbol{f}_{\text {vis }}, \boldsymbol{g}_{\text {vis }}$ can be found below,

$$
\boldsymbol{f}_{i n v}=\left(\begin{array}{c}
\rho u \\
\rho u^{2}+p \\
\rho u v \\
(E+p) u
\end{array}\right), \boldsymbol{g}_{i n v}=\left(\begin{array}{c}
\rho v \\
\rho u v \\
\rho v^{2}+p \\
(E+p) v
\end{array}\right)
$$




$$
\boldsymbol{f}_{v}=\left(\begin{array}{c}
0 \\
\tau_{x x} \\
\tau_{x y} \\
u \tau_{x x}+v \tau_{x y}+k \frac{\partial T}{\partial x}
\end{array}\right), \boldsymbol{g}_{v}=\left(\begin{array}{c}
0 \\
\tau_{x y} \\
\tau_{y y} \\
u \tau_{x y}+v \tau_{y y}+k \frac{\partial T}{\partial y}
\end{array}\right)
$$

Herein $k=\frac{\mu C_{p}}{\operatorname{Pr}}, \operatorname{Pr}=0.72$ and $\mu$ is also treated as a constant in the present work. Viscous stresses are given by

$$
\left\{\begin{array}{l}
\tau_{x x}=\mu\left(\frac{4}{3} \frac{\partial u}{\partial x}-\frac{2}{3} \frac{\partial v}{\partial y}\right) \\
\tau_{y y}=\mu\left(\frac{4}{3} \frac{\partial v}{\partial y}-\frac{2}{3} \frac{\partial u}{\partial x}\right) \\
\tau_{x y}=\tau_{y x}=\mu\left(\frac{\partial u}{\partial y}+\frac{\partial v}{\partial x}\right)
\end{array} .\right.
$$

At low Mach regions, the eigenvalues of the convection part of Eq. (1), i.e., $|\boldsymbol{v}|,|\boldsymbol{v}|+c$ and $|\boldsymbol{v}|-c$ are significantly different from each other. This disparity makes the hyperbolic system stiff to solve. Preconditioning methods replace the phycical acoustic wave speeds with numerical ones to decrease the condition number.

\section{Low Mach Number Preconditioning}

On using the chain-rule, Navier-Stokes equations can be written as

$$
\frac{\partial \boldsymbol{q}^{c}}{\partial \boldsymbol{q}^{p}} \frac{\partial \boldsymbol{q}^{p}}{\partial t}+\frac{\partial \boldsymbol{f}}{\partial x}+\frac{\partial \boldsymbol{g}}{\partial y}=0
$$

where $\boldsymbol{f}=\boldsymbol{f}_{\text {inv }}-\boldsymbol{f}_{\text {vis }}, \boldsymbol{g}=\boldsymbol{g}_{\text {inv }}-\boldsymbol{g}_{\text {vis }}, \boldsymbol{q}^{p}=(p, u, v, T)^{T}$ are the primitive variables, $\boldsymbol{M}=\frac{\partial \boldsymbol{q}^{c}}{\partial \boldsymbol{q}^{p}}$ is the Jacobian matrix between two sets of variables, i.e. conservative variables and primitive variables, which can be expressed as

$$
\boldsymbol{M}=\frac{\partial \boldsymbol{q}^{c}}{\partial \boldsymbol{q}^{p}}=\left(\begin{array}{cccc}
\rho_{p} & 0 & 0 & \rho_{T} \\
\rho_{p} u & \rho & 0 & \rho_{T} u \\
\rho_{p} v & 0 & \rho & \rho_{T} v \\
\rho_{p} H-1 & \rho u & \rho v & \rho_{T} H+\rho C_{p}
\end{array}\right)
$$

Herein, $H$ is the specific total enthalpy defined as $H=C_{p} T+\frac{1}{2}\left(u^{2}+v^{2}\right)$.

Recall the state equation $p=\rho R T$. Therefore, $\rho=\frac{p}{R T}$ and the derivatives in Eq. (6) can be calculated as ${ }^{17}$

$$
\rho_{p}=\left.\frac{\partial \rho}{\partial p}\right|_{T}=\frac{1}{R T}, \rho_{T}=\left.\frac{\partial \rho}{\partial T}\right|_{p}=-\frac{\rho}{T} .
$$

In the preconditioning method adopted by the present study, $\boldsymbol{M}$ is replaced with a matrix $\boldsymbol{\Gamma}$ called preconditioning matrix, which can cluster the eigenvalues of the hyperbolic system. The preconditioning matrix is given by

$$
\boldsymbol{\Gamma}=\left(\begin{array}{cccc}
\Theta & 0 & 0 & \rho_{T} \\
\Theta u & \rho & 0 & \rho_{T} u \\
\Theta v & 0 & \rho & \rho_{T} v \\
\Theta H-1 & \rho u & \rho v & \rho_{T} H+\rho C_{p}
\end{array}\right)
$$

In $\boldsymbol{M}, \rho_{p}=\frac{\partial \rho}{\partial p}$ is replaced by $\Theta$ as defined in Ref. 9

$$
\Theta=\left(\frac{1}{U_{r}^{2}}-\frac{\rho_{T}}{\rho C_{p}}\right)
$$


to get the preconditioning matrix $\boldsymbol{\Gamma}$. Herein, $U_{r}=\epsilon c$ and $\epsilon$ is a parameter related to the local Mach number $M a$ and the global cut-off Mach number. $c$ is the local speed of sound.

After introducing the preconditioning matrix into the compressible Navier-Stokes equations, the governing equations can be re-expressed as

$$
\boldsymbol{\Gamma} \frac{\partial \boldsymbol{q}^{p}}{\partial t}+\frac{\partial \boldsymbol{f}}{\partial x}+\frac{\partial \boldsymbol{g}}{\partial y}=0
$$

The preconditioned Jacobian matrix becomes $\boldsymbol{\Gamma}^{-1} \boldsymbol{A}_{\boldsymbol{n}}$ where $\boldsymbol{A}_{\boldsymbol{n}}=n_{x} \frac{\partial \boldsymbol{f}_{i n v}}{\partial \boldsymbol{q}^{p}}+n_{y} \frac{\partial \boldsymbol{g}_{i n v}}{\partial \boldsymbol{q}^{p}} . \quad \boldsymbol{n}=$ $\left(n_{x}, n_{y}\right)^{T}$ is the unit directional vector. The eigenvalues of this system are

$$
\left\{\begin{array}{l}
\lambda_{1}=\lambda_{2}=u \\
\lambda_{3}=u^{\prime}+c^{\prime} \\
\lambda_{4}=u^{\prime}-c^{\prime}
\end{array}\right.
$$

where

$$
\left\{\begin{array}{l}
u=\boldsymbol{v} \cdot \boldsymbol{n} \\
u^{\prime}=u(1-\alpha) \\
c^{\prime}=\sqrt{\alpha^{2} u^{2}+U_{r}^{2}} \\
\alpha=\left(1-\beta U_{r}^{2}\right) / 2 \\
\beta=\left(\rho_{p}+\frac{\rho_{T}}{\rho C_{p}}\right)
\end{array}\right.
$$

For an ideal gas, $\beta=(\gamma R T)^{-1}=1 / c^{2}$. At low speed, as $U_{r} \rightarrow 0, \alpha=\frac{1}{2}$. All the eigenvalues will have the same magnitude as $u$. Thus, the condition number of the original Navier-Stokes equations is significantly decreased.

It has been reported that preconditioning only based on local $M a$ through the entire domain will result in instabilities near the stagnation point and cause local pressure disturbances. ${ }^{8,10} \mathrm{~A}$ global cut-off parameter proportional to $M a_{\infty}{ }^{10,12}$ is usually used to prevent this robustness deterioration. Besides, for viscous flows, near the wall boundaries, the local flows are dominated by diffusion rather than convection. It is reasonable to release the preconditioning at these regions. ${ }^{18,28}$ In the present study, when no moving grids are involved, the parameter $\epsilon$ in $U_{r}=\epsilon c$ is defined as

$$
\epsilon=\min \left(1, \max \left(\alpha M a_{\infty}, M a\right)\right)
$$

where $\alpha$ is a free parameter which can be chosen based on different cases. Ref. 12 has provided suggestions for the values of $\alpha$.

The matrix $\boldsymbol{\Gamma}$ is treated as a local constant which definitely destroys the time accuracy of equation (5). Therefore, the approach described above can only be used for steady flow simulation. The GMRES solver with ILU0 preconditioner of PETSc library is used to solve Eq. (10).

When dynamic meshes is involved, the unsteady governing equation needs to be solved. Thus, after introducing the pseudo-time $\tau$, Eq. (10) can be rewritten as

$$
\frac{\partial \boldsymbol{q}^{c}}{\partial t}+\boldsymbol{\Gamma} \frac{\partial \boldsymbol{q}^{p}}{\partial \tau}+\frac{\partial \boldsymbol{f}}{\partial x}+\frac{\partial \boldsymbol{g}}{\partial y}-u_{g} \frac{\partial \boldsymbol{q}^{c}}{\partial x}-v_{g} \frac{\partial \boldsymbol{q}^{c}}{\partial y}=0
$$

The dual time stepping approach for unsteady flows will be discussed in the following section. In this section we only focus on the preconditioning algorithm. Note that grid velocity $\boldsymbol{v}_{g}=\left(u_{g}, v_{g}\right)$ are only related to the physical time $t$. Thus, when marching through the pseudo-time $\tau$, the grid velocity $\boldsymbol{v}$ can be regarded as a constant. Hence, the preconditioned Jacobian matrix for the 
convection terms becomes $\boldsymbol{\Gamma}^{-1} \boldsymbol{A}_{\boldsymbol{n}, \text { mov }}$, where $\boldsymbol{A}_{\boldsymbol{n}, \text { mov }}=n_{x} \frac{\partial \boldsymbol{f}_{\boldsymbol{i n v}}}{\partial \boldsymbol{q}^{p}}+n_{y} \frac{\partial \boldsymbol{g}_{i n v}}{\partial \boldsymbol{q}^{p}}-u_{g} \frac{\partial \boldsymbol{q}^{c}}{\partial x} n_{x}-v_{g} \frac{\partial \boldsymbol{q}^{c}}{\partial y} n_{y}$. The eigenvalues of this system preserve the same form as Eq. (11) and Eq. (12) with minor change, i.e., $u$ in Eq. (12) is modified to

$$
u=\left(\boldsymbol{v}-\boldsymbol{v}_{g}\right) \cdot \boldsymbol{n}
$$

due to the presence of the grid velocity. In spite of the fact that the grid velocity and flow velocity are coupled in the eigensystem, ${ }^{29}$ in the present study, we want to isolate the $M a$ number of the grid velocity from the flow velocity since the grid velocity is also an unsteady feature of the flow. ${ }^{30}$ The preconditioning parameter $\epsilon$ for dynamic mesh is simply defined as

$$
\epsilon=\min \left(1, \max \left(\alpha M a_{\infty}, M a, M a_{g}\right)\right),
$$

where $M a_{g}$ is the local $M a$ number of the grid velocity. This definition of $\epsilon$ will be tested using several numerical tests.

\section{Dual Time Stepping For Unsteady flows}

As stated previously, the preconditioning matrix $\boldsymbol{\Gamma}$ destroys the time accuracy of Eq. (5). Therefore, in order to handle unsteady flows, preconditioning will only work in pseudo-time iterations. Thus, the governing equation can be reformulated as Eq. (14) after introducing the pseudo-time $\tau$. Between two physical time iterations, the system is driven to converge in terms of pseudo-time iterations. Let $\boldsymbol{R}=\frac{\partial \boldsymbol{f}}{\partial x}+\frac{\partial \boldsymbol{g}}{\partial y}-u_{g} \frac{\partial \boldsymbol{q}^{c}}{\partial x}-v_{g} \frac{\partial \boldsymbol{q}^{c}}{\partial y}$ and on substituting it into Eq. (14), we have

$$
\boldsymbol{\Gamma} \frac{\partial \boldsymbol{q}^{p}}{\partial \tau}=-\frac{\partial \boldsymbol{q}^{c}}{\partial t}+\boldsymbol{R}
$$

In the discretized form, let $m$ denote current pseudo-time step and $n$ denote current physical-time step. Then we have

$$
\boldsymbol{\Gamma}^{m} \frac{\left(\boldsymbol{q}^{p}\right)^{m+1}-\left(\boldsymbol{q}^{p}\right)^{m}}{\Delta \tau}=-\left(\frac{\partial \boldsymbol{q}^{c}}{\partial t}\right)^{m+1}+\boldsymbol{R}^{m+1} .
$$

where $\left(\boldsymbol{q}^{p}\right)^{m=0}=\left(\boldsymbol{q}^{p}\right)^{n}$ and when $m \rightarrow \infty,\left(\boldsymbol{q}^{p}\right)^{m} \rightarrow\left(\boldsymbol{q}^{p}\right)^{n+1}$. At any time step, regardless of subscript ' $m$ ' and ' $\mathrm{n}$ ', the conservative variables can be updated by updating primitive variables. To achieve a second-order temporal accuracy, BDF2 is used. Thus, Eq. (18) is discretized as

$$
\boldsymbol{\Gamma}^{m} \frac{\left(\boldsymbol{q}^{p}\right)^{m+1}-\left(\boldsymbol{q}^{p}\right)^{m}}{\Delta \tau}=-\frac{3\left(\boldsymbol{q}^{c}\right)^{m+1}-4\left(\boldsymbol{q}^{c}\right)^{n}+\left(\boldsymbol{q}^{c}\right)^{n-1}}{2 \Delta t}+\boldsymbol{R}^{m+1}
$$

After linearizing $\boldsymbol{R}^{m+1}$ with respect to $\boldsymbol{R}^{m}$, Eq. (19) can be further written as

$$
\begin{aligned}
& \left(\frac{\boldsymbol{\Gamma}^{m}}{\Delta \tau}+\frac{3}{2 \Delta t} \boldsymbol{M}^{m}-\left(\frac{\partial \boldsymbol{R}}{\partial \boldsymbol{q}^{p}}\right)^{m}\right) \Delta\left(\boldsymbol{q}^{p}\right)^{m} \\
& =-\frac{3\left(\boldsymbol{q}^{c}\right)^{m}-4\left(\boldsymbol{q}^{c}\right)^{n}+\left(\boldsymbol{q}^{c}\right)^{n-1}}{2 \Delta t}+\boldsymbol{R}^{m} .
\end{aligned}
$$

where $\Delta\left(\boldsymbol{q}^{p}\right)^{m}=\left(\boldsymbol{q}^{p}\right)^{m+1}-\left(\boldsymbol{q}^{p}\right)^{m}$. In the present study, $\frac{\partial \boldsymbol{R}}{\partial \boldsymbol{q}^{p}}$ is computed numerically and only the block-diagonal terms are considered. Details of this approach can be found in Ref. 26 .

\section{Spatial Discretization}

The FR/CPR formulation is adopted to discretize Eq. (14) in space. In the current study, spatial domain is divided into non-overlapping quadrilateral elements. In FR/CPR, two sets of 
points contribute to the reconstructed polynomial, i.e. inner solution points(Gaussian points are selected in this study) and flux points on element interfaces. Fluxes can be divided into two parts, namely, local flux constructed directly from local solution points and correction flux reconstructed from flux points with correction functions. Common fluxes, i.e. fluxes at flux points, are obtained by Riemann solvers to guarantee conservation at element interfaces. Without considering the term involving physical time $t$ and viscous terms in Eq. (14), it can be reformulated as

$$
\boldsymbol{\Gamma} \frac{\partial \boldsymbol{q}^{p}}{\partial \tau}+\frac{\partial \boldsymbol{f}^{l o c}}{\partial x}+\frac{\partial \boldsymbol{g}^{l o c}}{\partial y}-u_{g} \frac{\partial \boldsymbol{q}^{c, l o c}}{\partial x}-v_{g} \frac{\partial \boldsymbol{q}^{c, l o c}}{\partial y}+\frac{1}{|\boldsymbol{J}|}\left(\frac{\partial \boldsymbol{F}^{c o r}}{\partial \xi}+\frac{\partial \boldsymbol{G}^{c o r}}{\partial \eta}\right)=0 .
$$

where

$$
\left\{\begin{array}{l}
\boldsymbol{F}=|\boldsymbol{J}|\left(\boldsymbol{q}^{c} \xi_{t}+\boldsymbol{f} \xi_{x}+\boldsymbol{g} \xi_{y}\right) \\
\boldsymbol{G}=|\boldsymbol{J}|\left(\boldsymbol{q}^{c} \eta_{t}+\boldsymbol{f} \eta_{x}+\boldsymbol{g} \eta_{y}\right)
\end{array}\right.
$$

and

$$
\boldsymbol{J}=\frac{\partial(x, y)}{\partial(\xi, \eta)},|\boldsymbol{J}|=x_{\xi} y_{\eta}-x_{\eta} y_{\xi}
$$

and

$$
\left\{\begin{array}{l}
\xi_{t}=-\boldsymbol{v}_{g} \cdot \nabla \xi \\
\eta_{t}=-\boldsymbol{v}_{g} \cdot \nabla \eta
\end{array}\right.
$$

Specifically, for quadrilateral elements, $F^{c o r}$ and $G^{c o r}$ are given by

$$
\left\{\begin{aligned}
\boldsymbol{F}^{c o r}(\xi, \eta) & =\left(\boldsymbol{F}_{L}^{\text {num }}(\eta)-\boldsymbol{F}^{l o c}(-1, \eta)\right) g_{L}^{c}(\xi) \\
& +\left(\boldsymbol{F}_{R}^{\text {num }}(\eta)-\boldsymbol{F}^{l o c}(1, \eta)\right) g_{R}^{c}(\xi), \\
\boldsymbol{G}^{c o r}(\xi, \eta) & =\left(\boldsymbol{G}_{L}^{\text {num }}(\xi)-\boldsymbol{G}^{l o c}(\xi,-1)\right) g_{L}^{c}(\eta) \\
& +\left(\boldsymbol{G}_{R}^{\text {num }}(\xi)-\boldsymbol{G}^{l o c}(\xi, 1)\right) g_{R}^{c}(\eta) .
\end{aligned}\right.
$$

Herein, subscripts ' $L$ ' and ' $R$ ' stand for left and right edges of an element in a dimension by dimension sense. $g_{L}^{c}, g_{R}^{c}$ are the corresponding correction functions which map the differences between numerical fluxes and local fluxes on element edges into solution points within the current element. In present work, $g_{L}^{c}, g_{R}^{c}$ are left and right Radau polynomials respectively, which recover the standard DG scheme. Different choices of correction function $g^{c}$ can be found in Huynh's papers. $^{22,23}$ At element interfaces, the flux in normal direction can be written as

$$
\boldsymbol{f}_{\boldsymbol{n}}=\boldsymbol{f} n_{x}+\boldsymbol{g} n_{y}-\left(\boldsymbol{v}_{g} \cdot \boldsymbol{n}\right) \boldsymbol{q}^{c}
$$

Note that common fluxes are always calculated in the physical domain, As a result, $\boldsymbol{F}^{n u m}$ and $G^{\text {num }}$ can be expresses as

$$
\left\{\begin{array}{l}
\boldsymbol{F}^{\text {num }}=|\boldsymbol{J}||\nabla \xi| \boldsymbol{f}_{\boldsymbol{n}}^{\text {com }} \operatorname{sign}(\boldsymbol{n} \cdot \nabla \xi), \\
\boldsymbol{G}^{\text {num }}=|\boldsymbol{J}||\nabla \eta| \boldsymbol{f}_{\boldsymbol{n}}^{\text {com }} \operatorname{sign}(\boldsymbol{n} \cdot \nabla \eta),
\end{array}\right.
$$

where $\boldsymbol{f}_{\boldsymbol{n}}^{c o m}$ is the common flux in $\boldsymbol{n}$ direction. $\boldsymbol{f}_{\boldsymbol{n}}^{\text {com }}$ is given as, taking Rusanov solver as an example,

$$
\boldsymbol{f}_{\boldsymbol{n}}^{c o m}=\frac{1}{2}\left(\boldsymbol{f}_{\boldsymbol{n}}^{c u r}+\boldsymbol{f}_{\boldsymbol{n}}^{n b}\right)-\frac{1}{2}|\lambda|_{\max } \widetilde{\boldsymbol{\Gamma}}\left(\boldsymbol{q}^{p, n b}-\boldsymbol{q}^{p, c u r}\right) .
$$


where $\widetilde{\boldsymbol{\Gamma}}$ is the local preconditioning matrix calculated by averaging primitive variables on elements egdes, and superscripts "cur" and "nb" denote the current element and neighboring element, respectively. $|\lambda|_{\max }$ is the maximun values of the absolute values of the eigenvalues. Preconditioning does not affect the viscous terms. For details about FR/CPR methods regarding the viscous terms, the readers are referred to Refs. 23, 26 .

\section{Boundary Conditions}

Let $q^{p, b}$ denote the primitive variables to be calculated at the boundaries and $q^{p,+}$ denote the primitive variables of the interior domain close to the boundaries and $\boldsymbol{q}^{p, \infty}$ for the free stream flow. In the present study, a set of non-refecting boundary conditions are employed for the far field for inviscid flow as reported in Ref. 11,17, defined as

$$
\begin{cases}p^{b}=p^{+}, \boldsymbol{v}^{b}=\boldsymbol{v}^{\infty}, T^{b}=T^{\infty}, & \text { for infow }, \\ p^{b}=p^{\infty}, \boldsymbol{v}^{b}=\boldsymbol{v}^{+}, T^{b}=T^{+}, & \text {for outflow. }\end{cases}
$$

For invscid slip wall, the $\boldsymbol{q}^{p, b}$ is enforced as

$$
p^{b}=p^{+}, u^{b}=u^{+}-2\left(\boldsymbol{v}^{+} \cdot \boldsymbol{n}\right) n_{x}, v^{b}=v^{+}-2\left(\boldsymbol{v}^{+} \cdot \boldsymbol{n}\right) n_{y}, T^{b}=T^{+} .
$$

For the viscous flow, at far-field boundaries, the $\boldsymbol{q}^{p, b}$ is fixed as $\boldsymbol{q}^{p, \infty}$ when the domain is large enough. For wall boundaries, an adiabatic wall boundary condition is enforced. Riemman solver is used to calculated the common flux in normal direction at all boundaries except wall boundaries. ${ }^{17}$

\section{Numerical Results}

Several benchmark tests have been conducted to verify the performance of the preconditioned FR/CPR method in this section. The $L_{2}$ error is used for the accuracy study. Specifically, for any solution variables $s$ on domain $\Omega$, the $L_{2}$ error is defined as

$$
\operatorname{Error}\left(s, L_{2}, \Omega\right)=\sqrt{\frac{\int_{\Omega}\left(s-s^{\text {exact }}\right)^{2} d V}{\int_{\Omega} d V}} .
$$

Rusanov solver is used through all the numerical tests. $P^{4}$ elements are used if curved boundaries are involved. The drag and lift coefficients are defined as

$$
C_{d}=\frac{\text { Drag }}{\frac{1}{2} \rho_{\infty} U_{\infty}^{2} A}, C_{l}=\frac{\text { Lift }}{\frac{1}{2} \rho_{\infty} U_{\infty}^{2} A}
$$

Due to the fact that when $M a$ number is very small, the variation of pressure is trivial compared to the pressure of inflow, the pressure are normalized for a better visualization following

$$
p_{\text {norm }}=\frac{p-p_{\min }}{p_{\max }-p_{\min }},
$$

where $p_{\min }$ and $p_{\max }$ are the minimum and maximun pressure in the entire flow field.

\section{VII.A. Inviscid Flow over the NACA0012 Airfoil}

Inviscid flow of $M a=10^{-3}$ and $0^{\circ}$ angle of attack (AOA) has been studied in this section. It is a benchmark test to validate the preconditoned solver's performance on low Mach number 


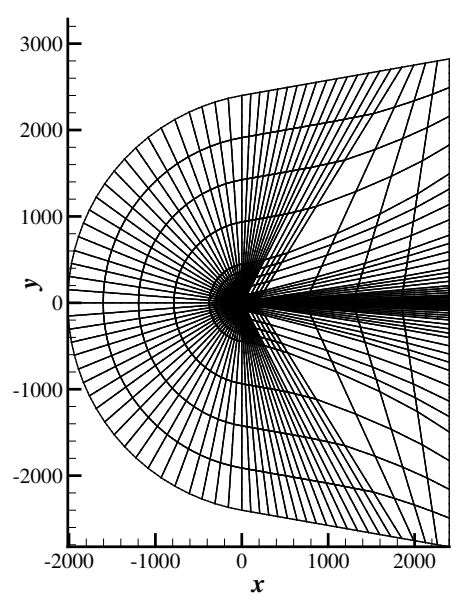

(a)

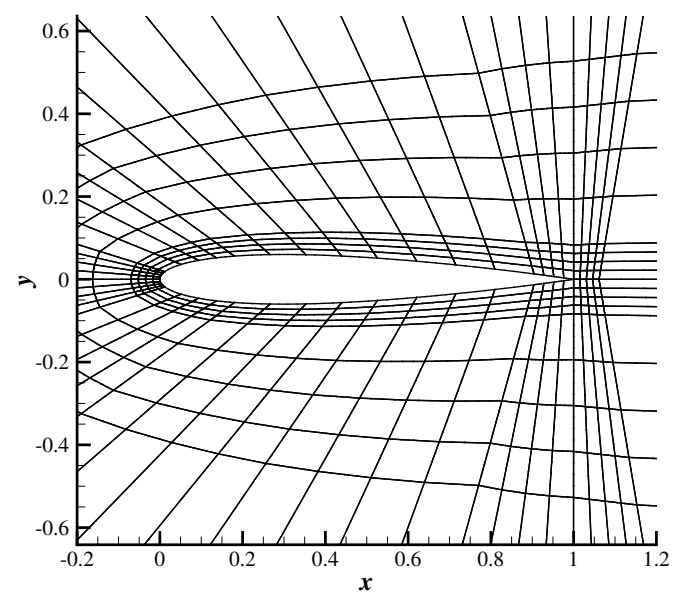

(b)

Figure 1. The domain and the 8960-element mesh, (a) an overview of the domain and the mesh; (b) the enlarged view of the mesh close to the airfoil.

flows. ${ }^{17}$ A mesh set with 560, 2240, 8960 elements $^{33}$ has been adopted for the grid refinement study of $3 r d, 4 t h, 5 t h \mathrm{FR} / \mathrm{CPR}$ methods. The finer mesh is refined by uniformly splitting the coarser mesh. An overview of the domain and mesh is illustrated in Figure 1.

In this study, the free parameter $\alpha$ in Eq. (13) is set as $\alpha^{2}=1.0$. Figure 2(a) illustrates the $M a$ contour of this case. In Figure 2(b), the normalized pressure contour near the airfoil region is presented. No pressure fluctuation is observed. From Figure 2(c), it is obvious that the preconditioned FR/CPR methods converges in only a few iterations and the residual of the pressure drops by almost 8 magnitudes. The grid refinement study of drag and lift coefficients are shown in Table 1. The abnormal increase of the $C_{d}$ and $C_{l}$ of the 5 th order FR/CPR method when the mesh is refined from 2240 elements to 8960 elements needs further study. In general, FR/CPR methods work well for this case.

Table 1. The grid refinement study of $C_{d}$ and $C_{l}$ of inviscid flow of $M a=10^{-3}$ and $A O A=2^{\circ}$ over the NACA0012 airfoil

\begin{tabular}{rcccccc}
\hline & \multicolumn{2}{c}{$P^{2}$} & \multicolumn{2}{c}{$P^{3}$} & \multicolumn{2}{c}{$P^{4}$} \\
\hline Elements & $C_{d}$ & $C_{l}$ & $C_{d}$ & $C_{l}$ & $C_{d}$ & $C_{l}$ \\
\hline 560 & $8.233 \times 10^{-3}$ & $1.016 \times 10^{-7}$ & $3.533 \times 10^{-3}$ & $1.024 \times 10^{-7}$ & $8.248 \times 10^{-4}$ & $3.953 \times 10^{-8}$ \\
2240 & $2.026 \times 10^{-3}$ & $1.788 \times 10^{-8}$ & $3.710 \times 10^{-4}$ & $3.528 \times 10^{-8}$ & $1.877 \times 10^{-5}$ & $7.536 \times 10^{-8}$ \\
8960 & $2.810 \times 10^{-4}$ & $8.619 \times 10^{-10}$ & $5.169 \times 10^{-6}$ & $1.250 \times 10^{-7}$ & $2.939 \times 10^{-5}$ & $1.731 \times 10^{-7}$ \\
\hline
\end{tabular}

\section{VII.B. Isentropic Vortex Propagation with Prescribed Grid Motion}

The isentropic vortex propagation case depicts the superposition of an inviscid uniform flow and an irrotational vortex. The vortex can be regarded as a perturbation added into the uniform flow. The free stream flow is of $(p, u, v, T)=(1.0, \sqrt{\gamma}, \sqrt{\gamma}, 1.0) . R=1.0$ for this case. The perturbation 


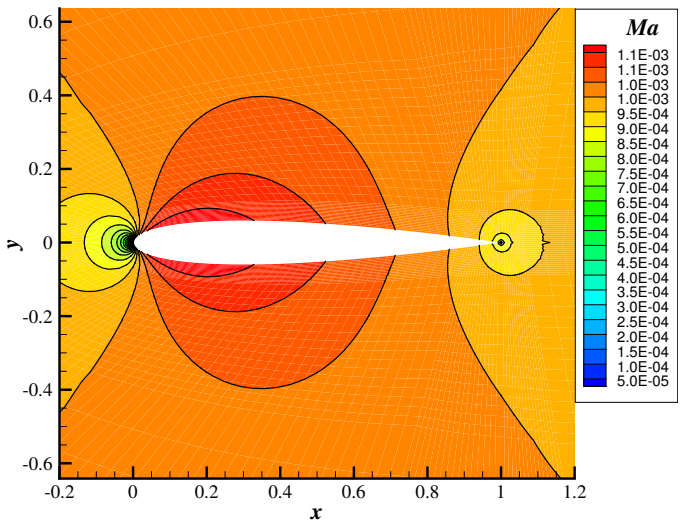

(a)

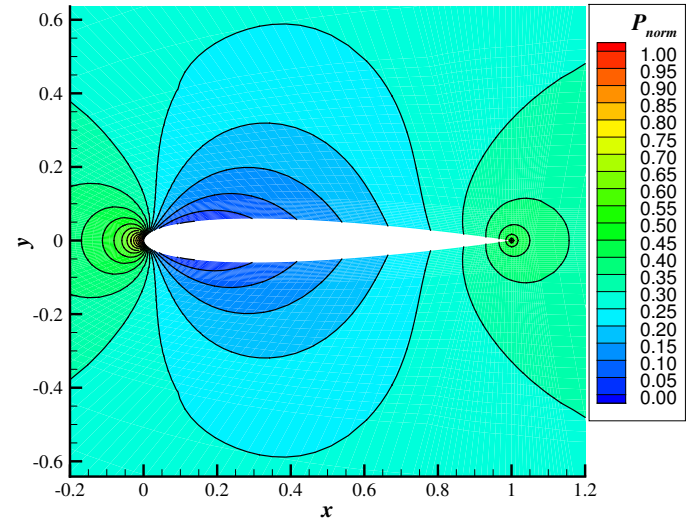

(b)

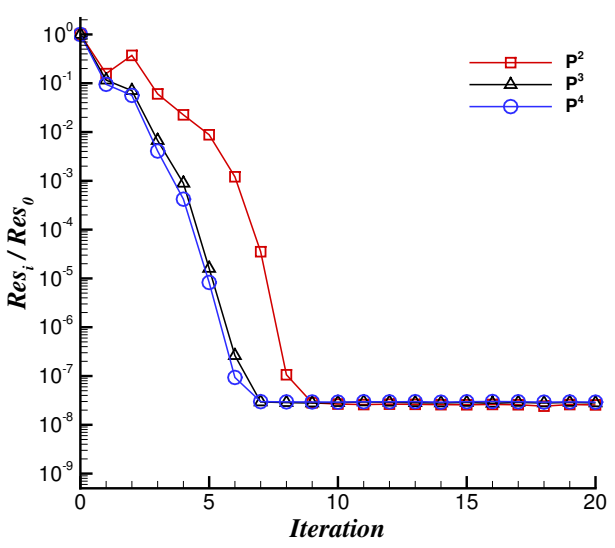

(c)

Figure 2. The inviscid flow of $M a=10^{-3}$ and $A O A=0^{\circ}$ over the NACA0012 airfoil, (a) $M a$ number contour of the 5 th order FR/CPR method close to airfoil on the 8960-element mesh(b) the normalized pressure contour of the 5 th order FR/CPR method on the 8960-element mesh; (c) Resi $/ R e s_{0}$ history of the $3 r d$, 4th and 5 th order FR/CPR methods. 


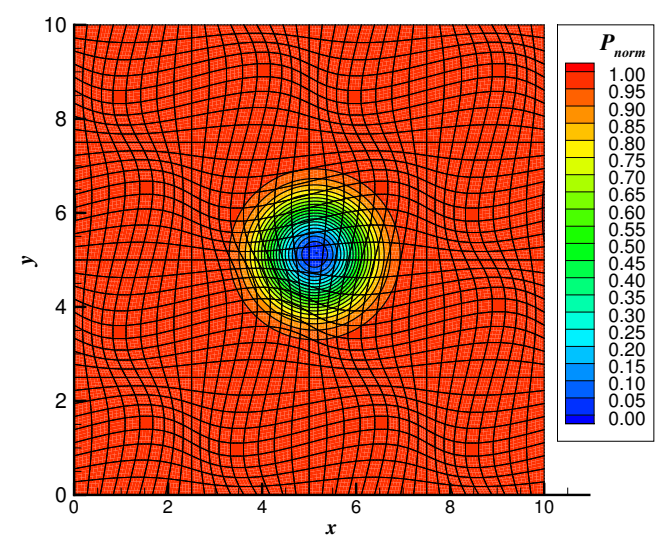

(a)

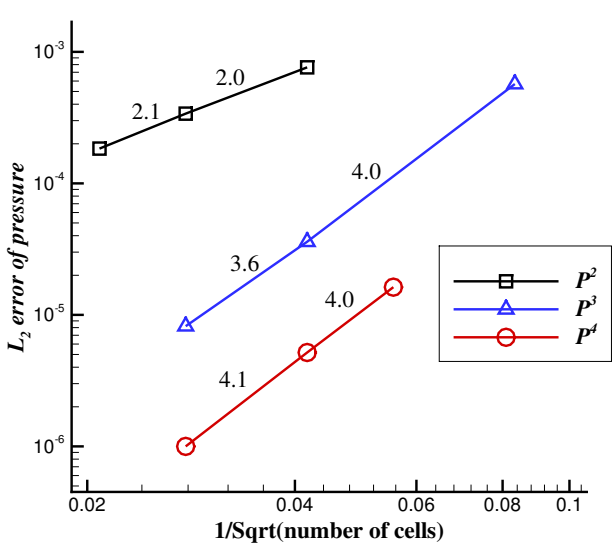

(b)

Figure 3. The isentropic vortex propagation results at $t=0.1$, (a) the normalized pressure contour on the $36 \times 36$ mesh; (b) the grid refinement study of the $3 r d, 4 t h, 5 t h$ FR/CPR methods.

is defined $\operatorname{as}^{20}$

$$
\left\{\begin{array}{l}
\delta u=-\frac{\alpha}{2 \pi}\left(y-y_{0}\right) e^{\phi\left(1-r^{2}\right)} \\
\delta v=\frac{\alpha}{2 \pi}\left(x-y_{0}\right) e^{\phi\left(1-r^{2}\right)} \\
\delta T=-\frac{\alpha^{2}(\gamma-1)}{16 \phi \gamma \pi^{2}}\left(y-y_{0}\right) e^{2 \phi\left(1-r^{2}\right)} \\
d S=0
\end{array}\right.
$$

where $\phi=\frac{1}{2}$ and $\alpha=5$ are parameters that define the vortex strength. $r=\left(x-x_{0}\right)^{2}+\left(y-y_{0}\right)^{2}$ is the distance to the center of the vortex $\left(x_{0}, y_{0}\right)=(5,5)$ at $t=0$. The periodic domain is defined in $[0,10] \times[0,10]$. A prescribed grid motion is employed in this case to validate the moving grid algorithm. The prescribed grid motion is defined as

$$
\left\{\begin{array}{l}
x(t)=x_{r}+a_{x} \sin \left(f_{n}(t)\right) \sin \left(f_{x} x_{r}\right) \sin \left(f_{y} y_{r}\right) \\
y(t)=y_{r}+a_{y} \sin \left(f_{n}(t)\right) \sin \left(f_{x} x_{r}\right) \sin \left(f_{y} y_{r}\right)
\end{array}\right.
$$

where $x_{r}, y_{r}$ are the coordinates at $t=0$, i.e., coordinates at the uniformly divided grid. $a_{x}=0.5$, $a_{y}=0.5, f_{n}=2.0 \pi, f_{x}=0.4 \pi, f_{y}=0.4 \pi$. A study of order of accuracy is carried out on a uniform mesh set, $12 \times 12,18 \times 18,24 \times 24,36 \times 36,48 \times 48$. Periodic boundary conditions are applied to all boundaries. Simulations stop at $t=0.1$. The time step size $\Delta t=10^{-5}$ and the free paramter in Eq. (16) is defined as $\alpha^{2}=1.0$. From Figure 3(a), it is observed that the FR/CPR methods with preconditioning can preserve the shape of the isentropic vortex well. The grid refinement study results are presented in Figure 3(b). The convergence rates agree well with theoretical values when Rusanov Riemann solver is used (i.e. $p+1 / 2$ ) despite of the difference between odd and even polynomial degree $p$.

\section{VII.C. Viscous Flow over a Stationary Circular Cylinder}

In this section, preconditioned FR/CPR methods are used to solve viscous flows over the circular cylinder. Both steady ${ }^{31}$ and unsteady ${ }^{26,32}$ flows over the stationary cylinder are tested, i.e. $R e=40$ and $R e=185$. The inflow is of $M a_{\infty}=10^{-3}$ and $u_{\infty}=1, v_{\infty}=0$. All the simulations are conducted on a $20 \times 24$ mesh. 20 is the number of elements in normal direction and 24 is the 
number of elements in circumstantial direction. An overview of the domain and mesh is presented in Figure 4.

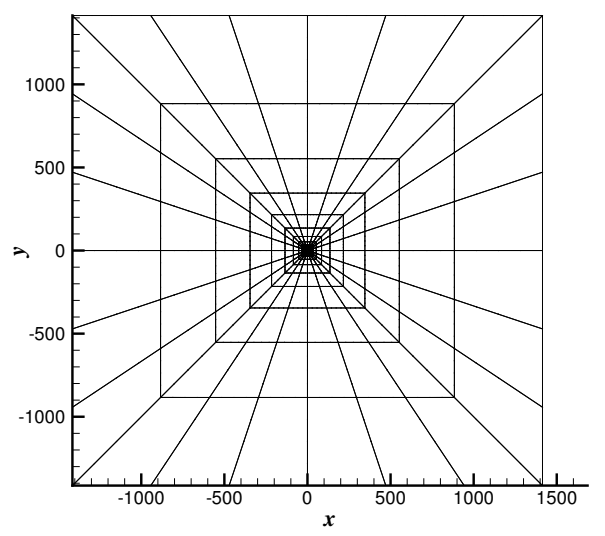

(a)

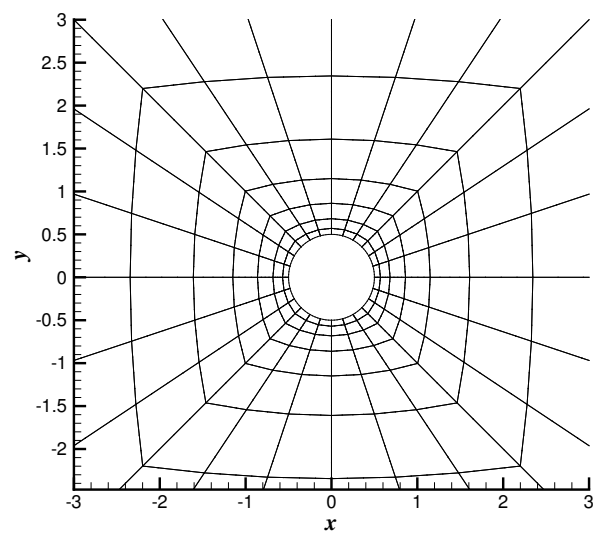

(b)

Figure 4. The domian and the $20 \times 24$ mesh, (a) an overview of the domain and the mesh; (b) the enlarged view of the mesh close to the cylinder.

When $R e=40$, the free parameter $\alpha$ in Eq. (13) is defined as $\alpha^{2}=1.0$. The normalized pressure contour of 5 th FR/CPR method when $R e=40$ is illustated in Figure 5. It is observed that the pressure contour is smooth near the wall boundary. A polynomial refinement from $3 \mathrm{rd}$ to 7 th order

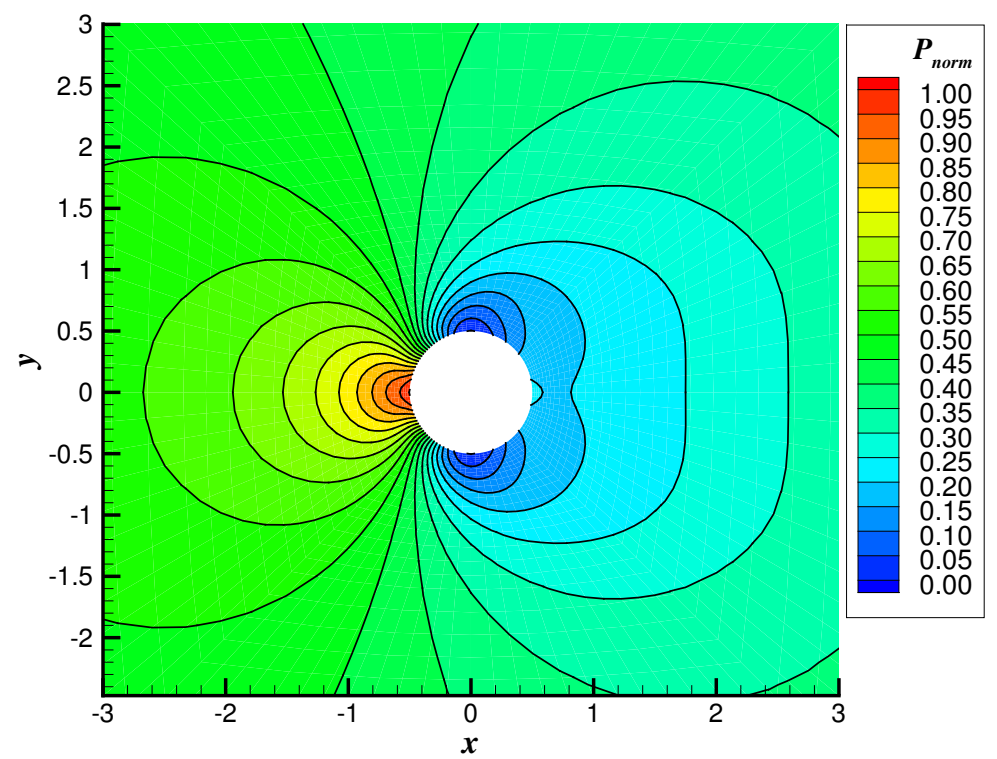

Figure 5. The normalized pressure contour of the flow of $M a=10^{-3}$ over the stationary circular cylinder when $R e=40$ on the $20 \times 24$ mesh using the 5 th FR/CPR method

FR/CPR methods is done when $R e=40$. The drag and lift coeffients of the polynomial refinement study are presented in Table 2 . From Table 2, it is observed that the $C_{l}$ keeps approximating 0 as the polynomial degree increases. And the drag coefficient agrees well with previous study.

For $R e=185$, a polynomial refinement study from $3 r d$ to 6 th order FR/CPR methods has been 
Table 2. The polynomial refinement study of $C_{d}$ and $C_{l}$ of the flow over the circular cylinder when Re=40

\begin{tabular}{rrrrrrr}
\hline & $3 r d$ & $4 t h$ & $5 t h$ & $6 t h$ & 7 th & Ref. 31 \\
\hline$C_{d}$ & 1.500 & 1.499 & 1.498 & 1.497 & 1.497 & 1.51 \\
$C_{l}$ & $4.836 \times 10^{-5}$ & $4.041 \times 10^{-6}$ & $1.266 \times 10^{-6}$ & $5.006 \times 10^{-7}$ & $2.081 \times 10^{-8}$ & - \\
\hline
\end{tabular}

conducted. The free parameter in Eq. (16) is defined as $\alpha^{2}=10$. Note that this makes the global cut-off value larger than any local $M a$ numbers, i.e., a global preconditioning parameter is chosen for the simulations. Time step size $\Delta t=0.01$. The results are presented in Table 3. Figure 6 is the

Table 3. The polynomial refinement study of $S t, \bar{C}_{d}$ and r.m.s. of $C_{l}$ of the flow over the circular cylinder when $\mathrm{Re}=185$

\begin{tabular}{rrrrrrr}
\hline & $3 r d$ & $4 t h$ & $5 t h$ & $6 t h$ & Ref. 26 & Ref. 32 \\
\hline$S t$ & 0.184 & 0.191 & 0.192 & 0.192 & 0.192 & 0.195 \\
\hline $\bar{C}_{d}$ & 1.261 & 1.315 & 1.317 & 1.318 & 1.317 & 1.31 \\
$C_{l, r m s}$ & 0.414 & 0.445 & 0.442 & 0.443 & 0.441 & 0.442 \\
\hline
\end{tabular}

force history of $C_{d}$ and $C_{l}$ in 100 seconds. The numerical results agree well with previous study.

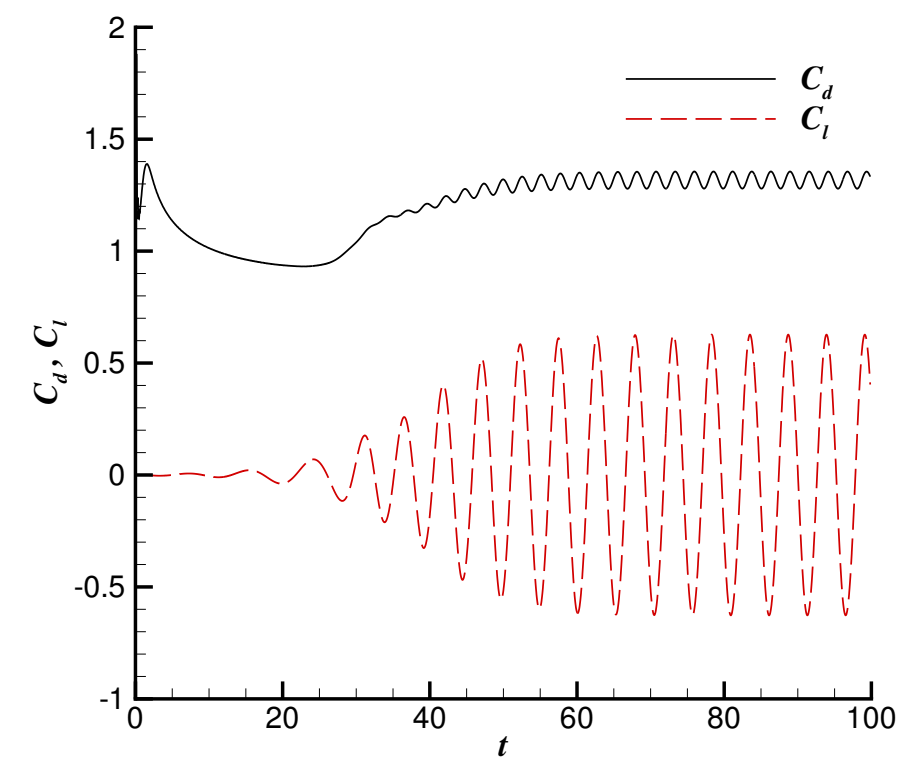

Figure 6. $C_{d}$ and $C_{l}$ history of flow over the stationary circular cylinder in 100 seconds when $R e=185$ using the 6 th order FR/CPR method

And results from Ref. 26 are obtained for FR/CPR methods with the artificial compressibility. Through the comparison of FR/CPR with different approaches to solve nearly incompressible flow, it is discovered that performances of FR/CPR methods with different approaches agree well each other. A snapshot of the vortex shedding is presented in Figure 7 of the 6 th order FR/CPR method. Even though the mesh is coarse, the $6 t h$ order FR/CPR method can still clearly capture the vortex shedding in wake region about 10 times of the diameter of the cylinder away from the cylinder center. 


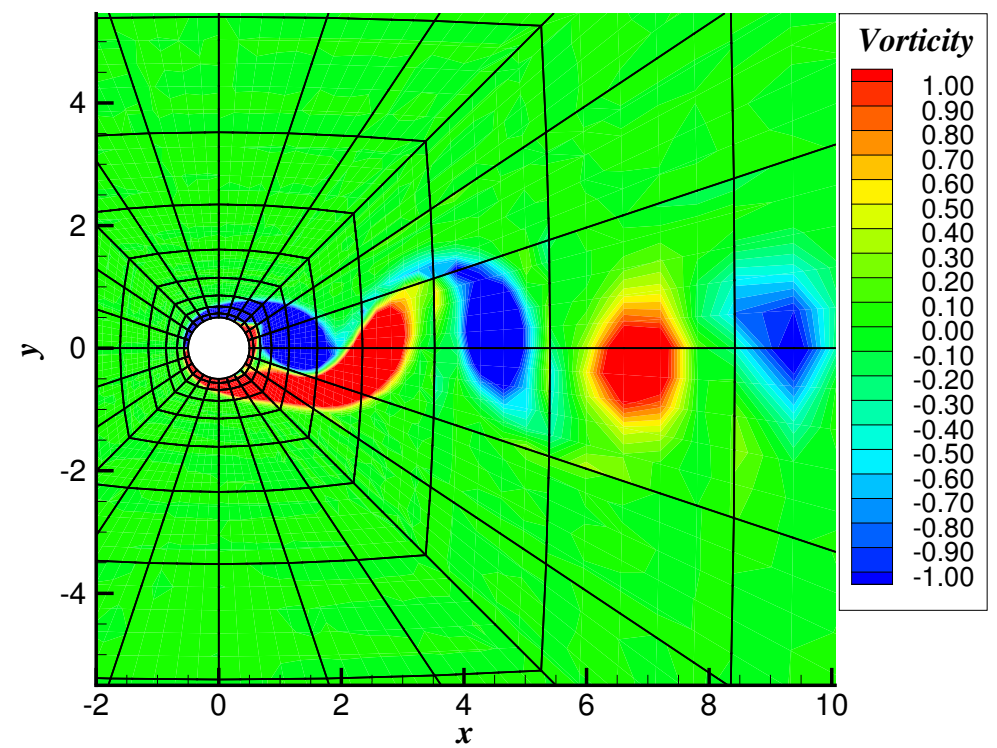

Figure 7. A snapshot of vorticity contour of flow over the stationary circular cylinder when $R e=185$, from -1 to 1,20 levels, 6th order FR/CPR method.

\section{VII.D. Viscous Flow over an Oscillating Circular Cylinder}

A prescribed motion of the cylinder in vertical direction (perpendicular to the flow direction of inflow) is employed to validate the performance of FR/CPR methods on dynamic meshes in this section. $^{26,32}$ The prescribed motion is defined as

$$
y=A_{e} \sin \left(2 \pi f_{e} t\right) .
$$

$R e=185$ is also studied in this section. The inflow conditions are the same as previous section. Frequency ratio $r=f_{e} / f_{0}$ is the ratio of oscillating frequency and natural frequency $f_{0} \cdot f_{0}=0.192$ obtained from previous section is used here. Different oscillating ratios are studied in this section, i.e., $r=0.8, r=1.0$ and $r=1.2$. The amplitude for this case is defined as $A_{e} / D=0.2$, where $D$ is the diameter of the cylinder. The time step size of all the simulations in this section is $\Delta t=0.01$. The free parameter in Eq. (16) is defined as $\alpha^{2}=5.0$. To conduct a fair comparison with Ref. 26. The 8 th order FR/CPR method is employed for the simulations. The $C_{d}$ and $C_{l}$ history of different oscillating frequency ratios are presented in Figure 8. And in Figure 8 we compare the results of the preconditioning method for low Mach number flows (LMP) and the artificial compressibility method (AC). From Figure 8(a,b), it is observed that the results from the 8 th order FR/CPR method with preconditioning agree well with the results from the $8 t h$ order FR/CPR method with the artificial compressibility. For $r \leq 1.0$, the vortex shedding process exhibits clearly periodic patterns. For $r>1.0$, chaotic features are observed in Figure 8(c). The wave packets of LMP and $\mathrm{AC}$ generally agree well with each other. It is also reasonable to discover the minor difference of some local pikes of the $C_{d}$ and $C_{l}$ in Figure 8(c) due to the highly unsteady and chaotic properties. 


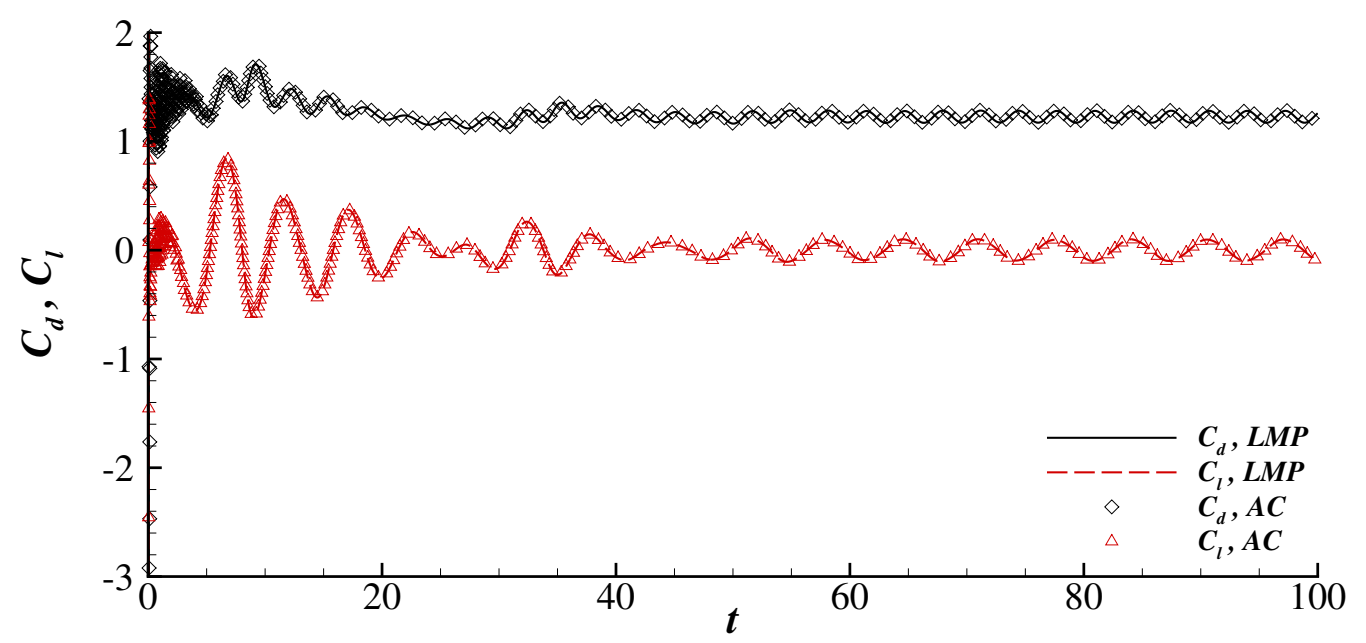

(a)

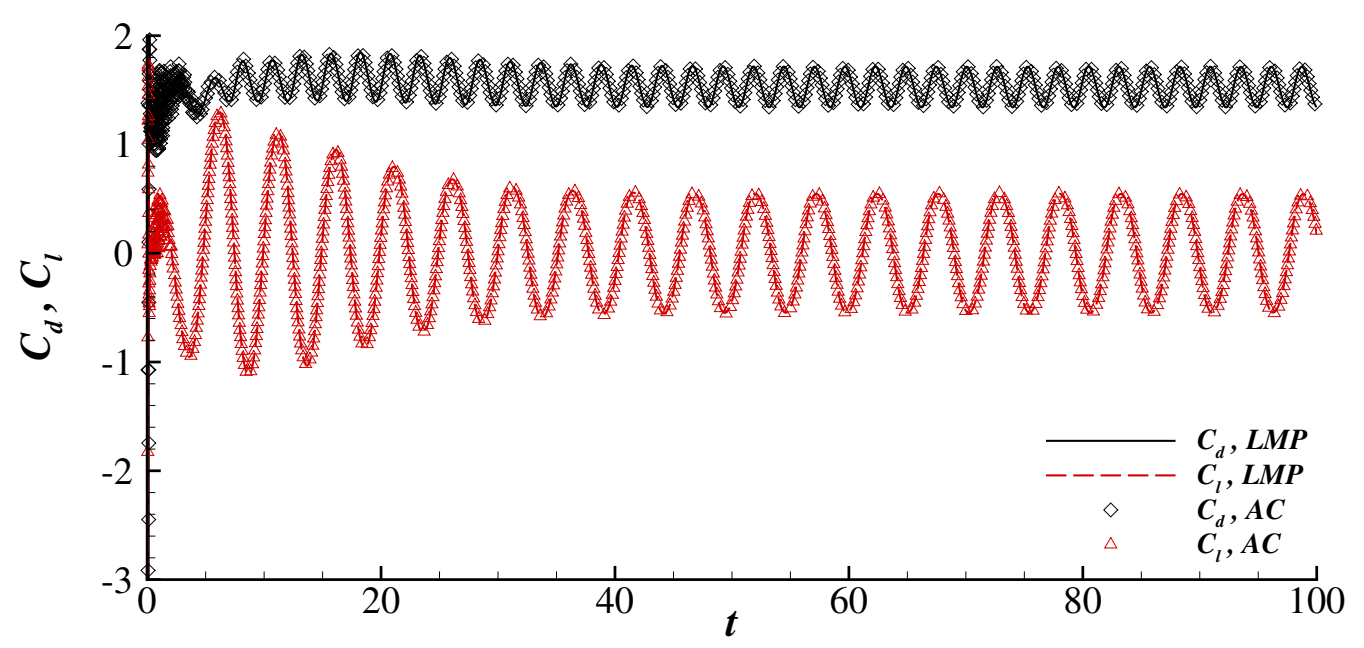

(b)

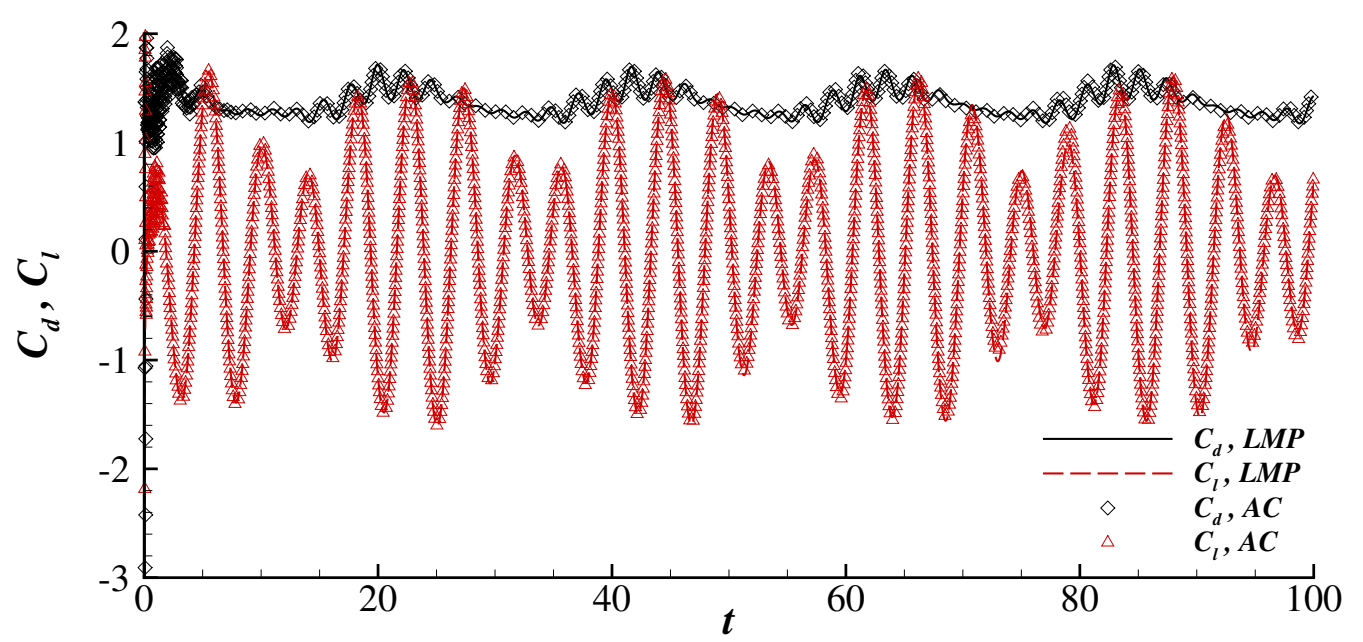

(c)

Figure 8. The $C_{d}$ and $C_{l}$ history of different oscillating frequency ratios $r$ using the 8 th FR/CPR metheod with the preconditioning method and the artificial compressibility method, (a) $r=0.8 ;$ (b) $r=1.0 ;$ (c) $r=1.2$. 


\section{Conclusions}

In the present study, the FR/CPR method is coupled with preconditioning method to solve compressible flow at low Mach number $\left(M a \sim \mathcal{O}\left(10^{-3}\right)\right)$ regions involving moving grids. The preconditioned FR/CPR methods proposed in the present study has been tested through several benchmark tests. Particularly, for the oscillating cylinder case, we compare the preconditioned FR/CPR method with the FR/CPR method using artificial compressibility for incompressible flows. Numerical results agree well with previous studies. The simple modification of the preconditioning formulation regarding grid velocities can efficiently handle the moving grids in the present study.

\section{Acknowledgement}

The authors gratefully acknowledge the support of the Office of Naval Research through the award N00014-16-1-2735, and the faculty startup support from the department of mechanical engineering at University of Maryland, Baltimore County (UMBC). 


\section{References}

${ }^{1}$ E. Turkel, A. Fiterman and B. Van Leer, "Preconditioning and the Limit to the Incompressible Flow Equations," NASA Contractor Report 191500, ICASE Report No. 93-42.

${ }^{2}$ L.S. Caretto, A.D. Gosman, S.V. Patankar and D.B. Spalding, "Two calculation procedures for steady, threedimensional flows with recirculation," Proceedings of the Third International Conference on Numerical Methods in Fluid Mechanics, Lecture Notes in Physics, Springer Berlin Heidelberg, pp. 60-68, 1997.

${ }^{3}$ S.V. Patankar, Numerical Heat Transfer and Fluid Flow, Hemisphere, Washington, D.C., 1980.

${ }^{4}$ A.J. Chorin, "Numerical solution of the Navier-Stokes equations." Mathematics of computation, vol. 22, iss. 104, pp. 745-762, 1968.

${ }^{5}$ A.J. Chorin, "A numerical method for solving incompressible viscous flow problems," Journal of computational physics, vol. 2, iss. 1,pp. 12-26, 1967.

${ }^{6}$ E. Turkel, "Preconditioned methods for solving the incompressible and low Speed compressible equations," Journal of Computational Physics, vol. 72, pp. 277-298, 1987.

${ }^{7}$ B. Van Leer, W.T. Lee and P.L. Roe, "Characteristic time-stepping or local preconditioning of the Euler equations," In 10th Computational Fluid Dynamics Conference, vol. 1, pp. 260-282, 1991.

${ }^{8}$ Y. H. Choi and C. L. Merkle, "The application of preconditioning in viscous flows," Journal of Computaional Physcics, vol. 105, pp. 207-233, 1993.

${ }^{9}$ J. M. Weiss and W. A. Smith, "Preconditioning applied to variable and constant density Flows," AIAA Journal, vol. 33, No. 11, 1995.

${ }^{10} \mathrm{~S}$. Venkateswaran and L. Merkle, "Analysis of preconditioning methods for the Euler and Navier-Stokes equations," Lecture series-van Kareman Institute for fluid dynamics 3, B1-B155, 1999.

${ }^{11}$ E. Turkel, R. Radespiel and N. Kroll, "Assessment of preconditioning methods for multidimensional aerodynamics," Computers $\&$ Fluids, vol. 26, No. 6, pp. 613-634, 1997.

${ }^{12}$ E. Turkel, "Preconditioning techniques in computational fluid dymamics," Annual Review of Fluid Mechanics, vol. 31, iss. 1, pp. 385-416, 1999.

${ }^{13}$ D. Lee and B. van Leer, "Progress in local preconditioning of the Euler and Navier-Stokes equations," $A I A A-$ 93-3328- $C P$.

${ }^{14} \mathrm{G}$. Hauke and T. Hughes, "A comparative study of different sets of variables for solving compressible and incompressible flows," Computer Methods in Applied Mehcanics and Engineering, vol. 153, pp. 1-44, 1998.

${ }^{15}$ T. Schneider, N. Botta, K. J. Geratz and R. Klein, "Extension of finite volume compressible flow solvers to multi-dimensional, variable density zero Mach number flows," Journal of Computational Physics, vol. 155, issue 2, pp. 248-286, 1991.

${ }^{16}$ D. Vigneron, G. Deliége and J. A. Essers, "Low mach number local preconditioning for unsteady viscous finite volumes simulations on 3D unstructured grids," ECCOMAS CFD 2006: Proceedings of the European Conference on Computational Fluid Dynamics, Egmond aan Zee, The Netherlands, September 5-8, 2006. Delft University of Technology; European Community on Computational Methods in Applied Sciences (ECCOMAS), 2006.

${ }^{17}$ F. Bassi, C. De Bartolo, R. Hartmann and A. Nigro, "A discontinuous Galerkin method for inviscid low Mach number flows," Journal of Computational Physics, vol. 228, pp. 3996-4011, 2009.

${ }^{18}$ Y. Colin, H. Deniau and J-F. Boussuge, "A robust low speed preconditioning formulation for viscous flow computations," Computers $\&$ Fluids, vol. 47, iss. 1, pp. 1-15, 2011.

${ }^{19}$ A. Nigro, C. De Bartolo, R. Hartmann and F. Bassi, "Discontinuous Galerkin solution of preconditioned Euler equations for very low Mach number flows," International journal for numerical methods in fluids, vol. 63, iss. 4, pp. 449-467, 2010.

${ }^{20}$ F. Bassi, L. Botti, A. Colombo, A Ghidoni and F. Massa, "Linearly implicit Rosenbrock-type Runge-Kutta schemes applied to the Discontinuous Galerkin solution of compressible and incompressible unsteady flows," Computers \&f Fluids, vol. 118, iss. 2, pp. 305-320.

${ }^{21}$ F. Bassi, L. Botti, A. Colombo, A. Crivellini, A. Ghidoni and F. Massa, "On the development of an implicit highorder Discontinuous Galerkin method for DNS and implicit LES of turbulent flows," European Journal of MechanicsB/Fluids, vol. 55, pp. 367-379, 2016.

${ }^{22}$ H.T. Huynh, "A flux reconstruction approach to high-Order schemes including discontinuous Galerkin methods," in 18th AIAA Computational Fluid Dynamics Conference, AIAA-2007-4079, Miami, FL, 2007.

${ }^{23}$ H.T. Huynh, "A reconstruction approach to high-order schemes including discontinuous Galerkin methods for diffusion," in 47th AIAA Aerospace Sciences Meeting including The New Horizons Forum and Aerospace, AIAA2009-403,Orlando, FL, 2009. 
${ }^{24}$ Z.J. Wang and H.Y. Gao, "A unifying lifting collocation penalty formulation including the discontinuous Galerkin, spectral volume/difference methods for conservation laws on mixed grids," Journal of Computational Physics, vol. 98, pp. 209-220, 2014.

${ }^{25}$ P.E. Vincent, P. Castonguay and A. Jameson, "A new class of high-order energy stable flux reconstruction schemes," Journal of Scientific Computation, vol. 47, no. 1, pp.50-72, 2011.

${ }^{26}$ M.L. Yu and L. Wang, "A high-order flux reconstruction/correction procedure via reconstruction formulation for unsteady incompressible flow on unstructured moving grids." Computers \& Fluids, vol. 139,iss. 5, pp. 161-173.

${ }^{27}$ C. Cox, C.L. Liang and M.W. Plesniak, "A flux reconstruction solver for unsteady incompressible viscous flow using artificial compressibility with implicit dual time stepping", in 54th AIAA Aerospace Sciences Meeting, AIAA SciTech, http://dx.doi.org/10.2514/6.2016-1827

${ }^{28} \mathrm{~S}$. Renda, "Discontinuous Galerkin methods for all speed flows," PhD thesis, 2013.

${ }^{29}$ T.H. Xiao, H.S. Ang and S.Z. Yu, "A preconditioned dual time-stepping procedure coupled with matrix-free LU-SGS scheme for unsteady low speed viscous flows with moving objects," International Journal of Computational Fluid Dynamics, vol. 21, no. 3-4, pp. 165-173, 2007.

${ }^{30}$ M.A. Potsdam, V. Sankaran and S.A. Pandya, "Unsteady low Mach preconditioning with application to rotorcraft flows," in 18th AIAA Computational Fluid Dynamics conference, AIAA Paper 4473, 2007.

${ }^{31}$ J. Park, K. Kwon K and H. Choi., "Numerical solutions of flow past a circular cylinder at Reynolds numbers up to 160," KSME international Journal, vol. 12, iss. 6, pp. 1200-1205, 1998.

${ }^{32}$ X. Y. Lu and C. Dalton, "Calculation of the timing of vortex formation from an oscillating cylinder," Journal of Fluids and Structures, vol. 10, iss. 5, pp. 527-541, 1996.

${ }^{33}$ The 1st International Workshop on High-Order CFD Methods, the 50th AIAA Aerospace Sciences Meeting, Nashville, TN, 2012. 